\title{
Outcomes of early invasive treatment strategy in elderly patients with non-ST elevation acute coronary syndromes Elena Conti ${ }^{a}$, Maria B. Musumeci ${ }^{a}$, Jasmine P. Desideri ${ }^{a}$, Martina Ventura ${ }^{b}$, Danilo Fusco ${ }^{b}$, Luigi Zezza ${ }^{a}$, Marco De Giusti ${ }^{a}$, Andrea Berni ${ }^{a}$, Pietro Francia ${ }^{a}$, Massimo Volpe ${ }^{\mathrm{a}, \mathrm{c}}$ and Camillo Autore ${ }^{\mathrm{a}}$
}

Background As benefits of revascularization in non-ST elevation acute coronary syndromes (NSTEACSs) in the elderly are still unproven, we sought to assess the association between invasive or conservative management of NSTEACS and short-, mid- and long-term mortality or composite outcome of all-cause mortality and myocardial infarction in a cohort of consecutive elderly patients.

Methods and Results Consecutive NSTEACS patients older than $\mathbf{7 5}$ years discharged between 2006 and 2010 from a single intensive cardiac care unit, and managed with invasive or conservative strategy according to available guidelines were retrospectively surveyed. By multivariate regression and sensitivity analysis, crude and adjusted mortality and composite outcome were estimated at prespecified time points of short-term (in-hospital or $\mathbf{3 0}$ days mortality), mid-term (T1: 31 days to 6 months), and long-term (T2: 31 days to 12 months). A total of 453 patients (median age 80 years, $47 \%$ men) were evaluated; 301 $(66.5 \%)$ underwent invasive treatment. Invasive was associated with significantly lower risk of short- [odds ratio (OR) 0.28 , 95\% confidence interval $(\mathrm{Cl}) 0.12-0.67$, $P=0.004$ ], mid- (OR 0.33, 95\% $\mathrm{Cl} 0.16-0.67, P=0.003)$ and long-term mortality (OR $0.34,95 \% \mathrm{Cl} 0.20-0.58, P<.0001$ ). Invasive strategy was also associated with nonsignificant lower short- (OR $0.55,95 \% \mathrm{Cl} 0.28-1.07, P=0.077$ ), and highly significant lower mid- (OR $0.52,95 \% \mathrm{Cl} 0.34-0.81$, $P=0.003$ ) and long-term adjusted cumulative composite outcome rate (OR $0.68,95 \% \mathrm{Cl} 0.46-0.98, P=0.004$ ).

Conclusion In NSTEACS elderly patients, invasive strategy is independently associated with lower short-, mid- and long-term mortality and composite outcome.

J Cardiovasc Med 2016, 17:736-743

Keywords: angioplasty, conservative treatment, elderly, invasive treatment, myocardial infarction

aDivision of Cardiology, Department of Clinical and Molecular Medicine, Sapienza University of Rome, Ospedale Sant'Andrea, 'Department of Epidemiology, Lazio Regional Health Service, Rome and 'IRCCS Neuromed, Pozzilli (IS), Italy

Correspondence to Camillo Autore, MD, Division of Cardiology, Department of Clinical and Molecular Medicine, Sapienza University of Rome, Ospedale Sant'Andrea, Via di Grottarossa 1035-1039, 00189, Rome, Italy Tel: +0039 06 33775098; fax: +003906 33775038; e-mail: camillo.autore@uniroma1.it

Received 2 September 2015 Revised 7 January 2016 Accepted 17 January 2016

showed that invasive strategy yields better survival free from a composite of all-cause mortality, nonfatal myocardial infarction (MI), disabling stroke and repeat hospitalizations, mainly driven by recurrent $\mathrm{MI}^{7,8}$ Conversely, a 10-year follow-up of real-world data of 2002-2004 years ${ }^{9}$ points toward higher event rate and lower potential benefit from percutaneous revascularization in elderly as compared with young people. In addition a recent reanalysis of CRUSADE cohort indicates that even though older patients treated at academic hospitals are more likely to receive inhospital revascularization than their counterparts admitted at nonacademic hospitals, they get only a modestly lower risk-adjusted 30-day and not improved risk-adjusted 1 -year mortality. ${ }^{10}$

Our study aims to retrospectively assess the association between invasive or conservative management of NSTEACS and short-, mid- and long-term mortality or composite outcome of all-cause mortality and MI in a cohort of consecutive elderly patients. 


\section{Methods}

We conducted a retrospective analysis of a monocentric cohort of patients 75 years or older with NSTEACS admitted to our ICCU across years 2006-2010. Patients' data retrieved from clinical charts, and imaging storing systems, were analyzed by multivariate regression analysis.

All admitted patients hereby consented to retrospective anonymized participation into clinical surveys, as approved and validated by an ethics committee and complying to Helsinki declaration.

\section{Study population}

Consecutive patients aged at least 75 years at admission with a diagnosis of NSTEACS were selected. Chronic renal failure (clearance less than $30 \mathrm{ml} / \mathrm{min}$ ), acute anemia (according to WHO definition), previous or current oncologic or cerebrovascular history were not considered exclusion criteria. Either type 1 or type 2 NSTEACS were included.

\section{Treatment strategy}

Invasive strategy was defined as angiography at the index admission, including emergency, urgent or delayed percutaneous coronary intervention (PCI) or surgery (coronary artery bypass graft surgery, CABG), according to coronary anatomy, physician and patient preference. Conservative treatment was defined as a medical only treatment during the index admission, with drug therapies recommended by current guidelines. ${ }^{11}$ During the study enrolment period comprised between 2006 and 2010, considerable variations in ACS treatment protocols and pharmaceutics were not adopted, with consistent patterns of care across time, so that a uniform referral pattern to conservative or invasive strategy was adopted by the same physicians for all patients involved.

\section{Outcome measures}

Primary endpoint was defined as all-cause mortality at the prespecified time points. We considered as prespecified time points: short-term - the index admission (in-hospital or 30-day mortality); mid-term - T1 (T1), defined as the time between 31 days to 6 months; and long-term T2 (T2), defined as the time between 31 days to 12 immonths. To avoid the impact of the events that occurred in the ACS acute phase, we excluded the first 30 days following the index event from the analysis of mid- and long-term outcome.

Major bleedings were defined as a drop in hemoglobin of at least $3 \mathrm{~g} / \mathrm{dl}$ and/or need for transfusion.

Secondary endpoint was defined as the cumulative rate of the composite outcome of all-cause mortality and MI at short-term (30 days), mid-term (6 months) and long-term (12 months). The first occurring event was the censored event.

\section{Statistics}

Crude and adjusted mortality and cumulative composite outcome rates were calculated. Multivariate regression analysis was used to assess the effect of strategy on mortality and composite outcome, adjusting for factors (age, sex and comorbidities) that could affect study outcomes. Risk factors potentially associated with outcomes were chosen among the conditions identified in the literature and from clinical judgment. Among those factors, age and sex were considered $a$ priori risk factors; the others were selected by a stepwise bootstrap procedure to assign an importance rank for predictors in regression. The variables introduced into the models were age, sex, admission creatinine clearance, ejection fraction (EF), hemoglobin and Killip classes, admission heart rate, blood pressure and cardiac arrest, ST deviation, peak troponin level, time from admission to PCI, albumin serum levels (albumin serum levels $<3 \mathrm{~g} / \mathrm{dl}$ were considered as a surrogate marker of frailty).

To estimate the odds ratios (ORs) of mortality and composite outcome at 30 days by strategy, a multivariate logistic regression was applied. Conversely, to evaluate mortality at $\mathrm{T} 1$ and $\mathrm{T} 2$ time intervals, and cumulative event rate of composite outcome at 6 and 12 months, Cox proportional hazard models were calculated by taking into account the amount of time for which an experimental unit contributed to the study, after verification of the proportionality assumption.

Effect modification by age classes, and specific conditions identified in the literature and from clinical judgment, was also tested, including categorization into spontaneous atherothrombotic or secondary to ischemic imbalance infarction as advised by current guidelines. ${ }^{11}$

A sensitivity analysis using genetic matching was performed to evaluate the robustness of our results: OR obtained from risk adjustment techniques were compared with those obtained after genetic matching. Genetic matching, a generalization of propensity score matching, is a method of multivariate matching, which uses a search algorithm to determine the weight of each covariate, improving balance among the individual covariates by searching over the space of distance metrics to find the best metric for optimizing covariate balance.

\section{Sample size}

Sample power of our study population was verified by comparing the patients treated with invasive $(n=301)$ and conservative $(n=152)$ strategies, whereas the ratio between the rates of the two groups was about $1-3$ for the outcomes of mortality and 1-2 for the composite endpoints, using an alpha of 0.05 . It resulted always higher than $95 \%$, except for the composite outcome at short-term, where it was found to be $61 \%$.

All statistical analyses were performed by using SAS Version 9.2 and R 2.15.1. 
Table 1 Patients' clinical characteristics by adopted strategy

\begin{tabular}{|c|c|c|c|c|}
\hline & $\begin{array}{l}\text { Total } \\
n=453\end{array}$ & $\begin{array}{l}\text { Invasive strategy } \\
\quad 301(66.5)\end{array}$ & $\begin{array}{c}\text { Conservative strategy } \\
152(33.5)\end{array}$ & $P$ \\
\hline \multicolumn{5}{|l|}{ Demographic characteristics } \\
\hline \multicolumn{5}{|l|}{ Age tertile $(n, \%)$} \\
\hline $74-79$ years & 204 & $164(54.5)$ & $40(26.3)$ & $<0.0001$ \\
\hline $80-84$ years & 150 & $100(33.2)$ & $50(32.9)$ & \\
\hline $85+$ years & 99 & $37(12.3)$ & $62(40.8)$ & \\
\hline $\operatorname{Men}(n, \%)$ & 213 & $159(52.8)$ & $52(34.2)$ & 0.001 \\
\hline Body mass index $\mathrm{kg} / \mathrm{m}^{2}($ mean $\pm \mathrm{SD})$ & 453 & $26.7 \pm 4.7$ & $25.6 \pm 5.1$ & 0.028 \\
\hline \multicolumn{5}{|l|}{ Risk factors } \\
\hline Smoking $(n, \%)$ & 46 & $12(7.9)$ & $34(11.3)$ & 0.015 \\
\hline Dyslipidemia $(n, \%)$ & 220 & $161(53.5)$ & $59(38.8)$ & 0.003 \\
\hline Hypertension $(n, \%)$ & 414 & $276(91.7)$ & $138(90.8)$ & 0.749 \\
\hline Diabetes $(n, \%)$ & 188 & $124(41.2)$ & $64(42.1)$ & 0.853 \\
\hline \multicolumn{5}{|l|}{ Biochemical risk profile } \\
\hline CKMB I peak value $\mathrm{ng} / \mathrm{ml}($ mean $\pm \mathrm{SD})$ & 453 & $33.0 \pm 54.5$ & $39.8 \pm 82.1$ & 0.353 \\
\hline Troponin I peak value $\mathrm{ng} / \mathrm{ml}($ mean $\pm \mathrm{SD}$ ) & 453 & $15.3 \pm 62.8$ & $14.1 \pm 35.9$ & 0.782 \\
\hline Troponin I admission value $\mathrm{ng} / \mathrm{ml}($ mean \pm SD) & 453 & $3.5 \pm 11.7$ & $4.5 \pm 13.4$ & 0.424 \\
\hline NT-proBNP (mean \pm SD) & 453 & $6402 \pm 9878$ & $14524 \pm 16409$ & $<0.0001$ \\
\hline Total cholesterol mg/dl (mean $\pm \mathrm{SD})$ & 453 & $166 \pm 43$ & $163 \pm 39$ & 0.386 \\
\hline $\mathrm{HDL}$ cholesterol $($ mean $\pm \mathrm{SD})$ & 453 & $43 \pm 13$ & $43 \pm 13$ & 0.999 \\
\hline Triglycerides $($ mean $\pm \mathrm{SD})$ & 453 & $119 \pm 53$ & $125 \pm 68$ & 0.329 \\
\hline Admission glycemia mg/dl (mean $\pm \mathrm{SD})$ & 453 & $139 \pm 68$ & $165 \pm 85$ & 0.001 \\
\hline Admission albumin $\mathrm{mg} / \mathrm{dl}($ mean $\pm \mathrm{SD})$ & 453 & $3.4 \pm 0.46$ & $3.2 \pm 0.56$ & 0.002 \\
\hline \multicolumn{5}{|l|}{ Admission creatinine clearance classes } \\
\hline$>60 \mathrm{ml} / \min (n, \%)$ & 98 & $80(26.6)$ & $18(11.8)$ & $<0.0001$ \\
\hline $30-60 \mathrm{ml} / \mathrm{min}(n, \%)$ & 247 & $168(55.8)$ & $79(52.0)$ & \\
\hline$<30 \mathrm{ml} / \min (n, \%)$ & 99 & $49(16.3)$ & $50(32.9)$ & \\
\hline \multicolumn{5}{|l|}{ Admission hemoglobin quartiles } \\
\hline $1(5.7-11.1 \mathrm{~g} / \mathrm{dl})(\mathrm{n}, \%)$ & 129 & $59(19.6)$ & $70(46.1)$ & $<0.0001$ \\
\hline $2(11.2-12.45 \mathrm{~g} / \mathrm{dl})(n, \%)$ & 120 & $85(28.2)$ & $35(23.0)$ & \\
\hline $3(12.45-13.9)(n, \%)$ & 103 & $81(26.9)$ & $22(14.5)$ & \\
\hline $4(13.9-21)(n, \%)$ & 101 & $76(25.3)$ & $25(16.5)$ & \\
\hline \multicolumn{5}{|l|}{ Clinical risk profile } \\
\hline Admission SBP mmHg (mean \pm SD) (PAS) & 453 & $145 \pm 32$ & $143 \pm 31$ & 0.518 \\
\hline Admission heart rate bpm (mean \pm SD) & 453 & $85 \pm 24$ & $92 \pm 23$ & 0.005 \\
\hline Aspirin prescription in the previous 7 days $(n, \%)$ & & $138(45.8)$ & $72(47.4)$ & 0.759 \\
\hline $\mathrm{PCl}$ in the previous 30 days $(n, \%)$ & 4 & $2(0.7)$ & $2(1.3)$ & 0.484 \\
\hline CABG in the previous 30 days $(n, \%)$ & 0 & - & - & - \\
\hline \multicolumn{5}{|l|}{ Admission GRACE risk score classes } \\
\hline$\leq 150(n, \%)$ & 54 & $42(14.0)$ & $12(7.9)$ & 0.123 \\
\hline $150-170(n, \%)$ & 56 & $39(13.0)$ & $17(11.2)$ & \\
\hline$\geq 170(n, \%)$ & 343 & $220(73.1)$ & $123(80.9)$ & \\
\hline \multicolumn{5}{|l|}{ Killip class } \\
\hline $1(n, \%)$ & 215 & $160(53.2)$ & $55(36.2)$ & 0.002 \\
\hline $2(n, \%)$ & 181 & $112(37.2)$ & $69(45.4)$ & \\
\hline $3(n, \%)$ & 56 & $29(9.6)$ & $27(17.8)$ & \\
\hline $4(n, \%)$ & 1 & 0 & $1(0.7)$ & \\
\hline Cardiac arrest at admission $(n, \%)$ & 5 & $4(1.3)$ & $1(0.7)$ & 0.629 \\
\hline Max ST segment deviation & 453 & $85 \pm 24$ & $92 \pm 23$ & \\
\hline Ejection fraction classes & & & & 0.406 \\
\hline$>50 \%(n, \%)$ & 157 & $106(35.2)$ & $51(33.6)$ & 0.161 \\
\hline $30-50 \%(n, \%)$ & 227 & $156(51.8)$ & $71(46.7)$ & \\
\hline$<30 \%(n, \%)$ & 69 & $39(13.0)$ & $30(19.7)$ & \\
\hline Valvular heart disease ${ }^{\mathrm{a}}(\geq$ moderate) $(n, \%)$ & 240 & $149(49.5)$ & $91(59.9)$ & 0.037 \\
\hline \multicolumn{5}{|l|}{ Presentation symptoms } \\
\hline Chest pain $(n, \%)$ & 331 & $246(81.7)$ & 85 (55.9) & $<0.0001$ \\
\hline Dyspnea $(n, \%)$ & 242 & $144(47.8)$ & $98(64.5)$ & 0.001 \\
\hline Syncope $(n, \%)$ & 30 & $16(5.3)$ & $14(9.2)$ & 0.116 \\
\hline
\end{tabular}

CABG, coronary artery bypass graft surgery; CKMB, creatine kinase myocardial band; HDL, high-density lipoprotein; NT-proBNP, NT-pro Brain Natriuretic Peptide; PCl, percutaneous coronary intervention.

\section{Results}

\section{Baseline characteristics}

A total of 453 white patients (median age: 80 years, $47 \%$ men) with NSTEACS were evaluated. Of these, 301 $(66.5 \%)$ were treated with an invasive strategy, whereas in $152(33.5 \%)$ a conservative strategy was adopted. In the invasive group, 178 (59.1\%) patients underwent PCI and $14(4.7 \%)$ patients were treated with CABG. The remaining 109 patients (36.2\%) did not undergo revascularization because coronary lesions were considered nonsignificant $(n=51,46.8 \%)$ or not amenable by either percutaneous or surgical procedure $(n=50$, $45.9 \%)$. In eight patients $(7.3 \%)$ periprocedural risk was judged excessive and unacceptable. Major bleeding and/or need for transfusions occurred in 29 of patients who underwent invasive strategy: 14 out of $178(7.8 \%)$ 
Table 2 Patients' outcome according to invasive or conservative strategy

\begin{tabular}{lccc}
\hline & $\begin{array}{c}\text { Invasive } \\
n(\%) \\
301(66.5)\end{array}$ & $\begin{array}{c}\text { Conservative } \\
n(\%) \\
152(33.6)\end{array}$ & \\
& & & \\
& & & \\
Study endpoint & $8(2.7)$ & $14(9.2)$ & 0.002 \\
Mortality & $11(3.7)$ & $21(13.8)$ & $<0.0001$ \\
$\quad$ In-hospital & $17(5.9)$ & $23(17.6)$ & $<0.0001$ \\
30-Day cumulative & $29(10.4)$ & $36(27.5)$ & $<0.0001$ \\
T1 time point & $40(13.3)$ & $57(37.5)$ & $<0.0001$ \\
T2 time point & & & \\
12-Month cumulative & $25(8.3)$ & $24(15.8)$ & 0.016 \\
Cumulative composite outcome & $52(17.3)$ & $56(36.8)$ & $<0.0001$ \\
30-Day & $74(24.6)$ & $64(42.1)$ & $<0.0001$ \\
6-Month & $14(4.7)$ & $4(2.6)$ & \\
12-Month & $27(9.0)$ & $15(9.9)$ & 0.299 \\
AMI/ACS & $39(13.0)$ & $16(10.5)$ & 0.756 \\
30-Day AMI/ACS & & \\
6-Month AMI/ACS & & \\
12-Month AMI/ACS & $64(21.3)$ & $28(18.5)$ & 0.498 \\
Adverse clinical events during hospitalization & $36(23.7)$ & $<0.0001$ \\
Cardiac arrhythmia & $29(9.6)$ & $30(19.7)$ & $<0.0001$ \\
Major bleeding/transfusion & $20(6.6)$ & $12(8.0)$ & 0.050 \\
$\quad$ Sepsis/infections & $11(3.7)$ & & \\
AKI, whichever defined & &
\end{tabular}

AKI, acute kidney injury; AMI/ACS, acute myocardial infarction/acute coronary syndromes; T1, 31 days to 6 months; T2, 31 days to 12 months.

patients treated with PCI, 10 out of 14 patients (71.4\%) in the CABG group and finally 4 out of 109 patients (3.6\%) who had only coronary angiography.

Clinical and biochemical characteristics of invasive and conservative patients are reported in Table 1 . Invasive patients were more commonly men and younger than conservative patients $(P=0.001$ and $P<0.0001$, respectively). Median time from admission to procedure was low, equal to 1 day (interquantile range $0-3$ days), consistently with an early invasive strategy. Comorbidity profile was less severe in invasive patients than in conservative patients, as proved by the higher creatinine clearance and hemoglobin values (Table 1). Compared with conservative patients, invasive patients presented more often with classical ACS symptoms as chest pain (81.7 vs. $55.9 \%, P<0.001)$, while they had dyspnea or other symptoms less frequently (47.8 vs. $64.5 \%$, $P=0.001)$. Finally, invasive patients had lower Killip classes than conservative patients (class 1-2: 90.4 vs. $81.6 \%$, class $3-4: 9.6$ vs. $18.5 \%, P=0.002$, respectively) (Table 1).

\section{Outcome}

\section{Mortality}

A total of $40(13.3 \%)$ invasive patients and 57 (37.5\%) conservative patients died during 1-year follow-up $(P<0.0001)$. Mortality rates at the prespecified time points are reported in Table 2.

Crude and adjusted ORs to estimate the risk of mortality between strategies are reported in Table 3. Comparing adjusted short-term mortality in invasive to conservative group, a statistically significant reduction in 30-day mortality was observed in the invasive group [OR 0.28 , $95 \%$ confidence interval (CI) $0.12-0.67, P=0.004]$. This reduction was confirmed throughout the mid- and longterm follow-up at T1 (OR $0.33,95 \%$ CI $0.16-0.67$, $P=0.003$ ) and $\mathrm{T} 2$ periods (OR 0.34 , 95\% CI 0.20 $0.58, P<.0001$ ) (Fig. 1 A-C; Table 3). No interaction between the three defined age classes of young old, old,

Table 3 Death predictors at the prespecified time points by univariate and multivariate analysis

\begin{tabular}{|c|c|c|c|c|c|c|}
\hline Mortality & OR univariate & $95 \% \mathrm{Cl}$ & $P$ & OR multivariate & $95 \% \mathrm{Cl}$ & $P$ \\
\hline \multicolumn{7}{|l|}{ In-hospital } \\
\hline Invasive strategy ${ }^{a}$ & 0.27 & $0.11-0.66$ & 0.004 & 0.37 & $0.22-1.68$ & 0.060 \\
\hline Admission creatinine clearance $30-60 \mathrm{ml} / \mathrm{min}$ & 4.09 & $0.52-32.41$ & 0.182 & 4.21 & $0.51-35.05$ & 0.183 \\
\hline Admission creatinine clearance $<30 \mathrm{ml} / \mathrm{min}^{\mathrm{b}}$ & 10.90 & $1.37-86.87$ & 0.024 & 7.75 & $0.87-68.62$ & 0.066 \\
\hline Killip class $2^{c}$ & 4.13 & $1.12-15.25$ & 0.033 & 3.26 & $0.86-12.32$ & 0.082 \\
\hline Killip class $3^{c}$ & 13.25 & $3.46-50.79$ & 0.000 & 8.40 & $2.03-34.80$ & 0.003 \\
\hline \multicolumn{7}{|l|}{ 30-Day } \\
\hline Invasive strategy ${ }^{a}$ & 0.24 & $0.11-0.51$ & 0.000 & 0.28 & $0.12-0.67$ & 0.004 \\
\hline Ejection fraction $30-50 \%{ }^{d}$ & 1.85 & $0.64-5.29$ & 0.253 & 1.13 & $0.36-3.60$ & 0.830 \\
\hline Ejection fraction $\leq 30 \%{ }^{d}$ & 7.74 & $2.66-22.48$ & 0.000 & 3.40 & $1.00-11.58$ & 0.051 \\
\hline Admission Killip class $2^{\mathrm{e}}$ & 2.92 & $1.10-7.76$ & 0.032 & 1.90 & $0.66-5.45$ & 0.233 \\
\hline Admission Killip class $3^{\mathrm{e}}$ & 9.29 & $3.31-26.06$ & $<.0001$ & 4.64 & $1.45-14.88$ & 0.010 \\
\hline \multicolumn{7}{|l|}{ T1 Time Point } \\
\hline Invasive strategy ${ }^{a}$ & 0.31 & $0.17-0.58$ & 0.000 & 0.33 & $0.16-0.67$ & 0.003 \\
\hline Admission albumin level $>3 \mathrm{~g} / \mathrm{dl}$ & 0.26 & $0.14-0.50$ & $<.0001$ & 0.37 & $0.19-0.73$ & 0.004 \\
\hline Admission systolic blood pressure $(\mathrm{mmHg})$ & 0.99 & $0.98-1.00$ & 0.016 & 0.99 & $0.97-1.00$ & 0.011 \\
\hline Admission Killip class $2^{\mathrm{C}}$ & 3.62 & $1.61-8.14$ & 0.002 & 4.61 & $1.79-11.87$ & 0.002 \\
\hline Admission Killip class $3^{\mathrm{c}}$ & 6.17 & $2.44-15.64$ & 0.000 & 10.05 & $3.36-30.08$ & $<.0001$ \\
\hline Admission cardiac arrest episode & 4.87 & $0.67-35.49$ & 0.118 & 11.14 & $1.29-96.51$ & 0.029 \\
\hline \multicolumn{7}{|l|}{ T2 Time Point } \\
\hline Invasive strategy ${ }^{a}$ & 0.33 & $0.20-0.53$ & $<0.0001$ & 0.34 & $0.20-0.58$ & $<.0001$ \\
\hline Admission albumin level $>3 \mathrm{~g} / \mathrm{dl}$ & 0.37 & $0.22-0.60$ & $<0.0001$ & 0.46 & $0.27-0.78$ & 0.004 \\
\hline Admission systolic blood pressure $(\mathrm{mmHg})$ & 0.99 & $0.98-1.00$ & 0.017 & 0.99 & $0.98-1.00$ & 0.013 \\
\hline Diabetes & 2.04 & $1.26-3.37$ & 0.004 & 1.77 & $1.05-2.99$ & 0.031 \\
\hline Admission Killip class $2^{\mathrm{c}}$ & 2.29 & $1.30-4.00$ & 0.004 & 2.11 & $1.15-3.85$ & 0.002 \\
\hline Admission Killip class $3^{c}$ & 3.90 & $1.96-7.88$ & 0.000 & 3.96 & $1.86-8.44$ & $<0.0001$ \\
\hline
\end{tabular}

$\mathrm{Cl}$, confidence interval; OR, odds ratio. ${ }^{a}$ Conservative strategy as ref. ${ }^{b}>60 \mathrm{ml} / \mathrm{min}$ as ref. ${ }^{\mathrm{c}}$ Killip 1 as ref. ${ }^{d} \mathrm{EF}>50 \%$ as ref. ${ }^{e}$ Killip 1 as ref. 
Fig. 1

(a)

Death

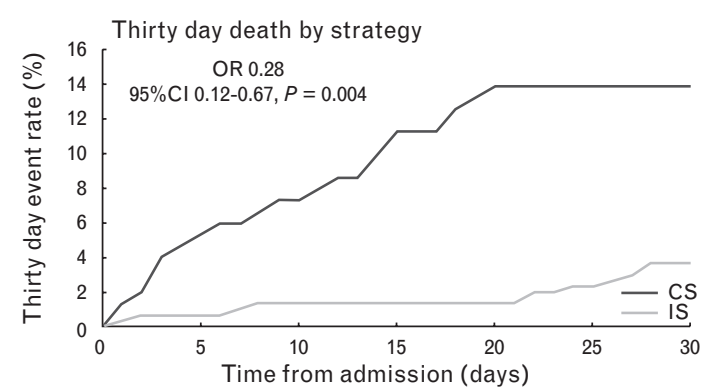

Strategy Number of patients at risk

$\begin{array}{llllllll}\text { IS } & 152 & 144 & 141 & 135 & 131 & 131 & 131\end{array}$

$\begin{array}{llllllll}\text { CS } & 301 & 299 & 297 & 297 & 297 & 294 & 290\end{array}$

(b)

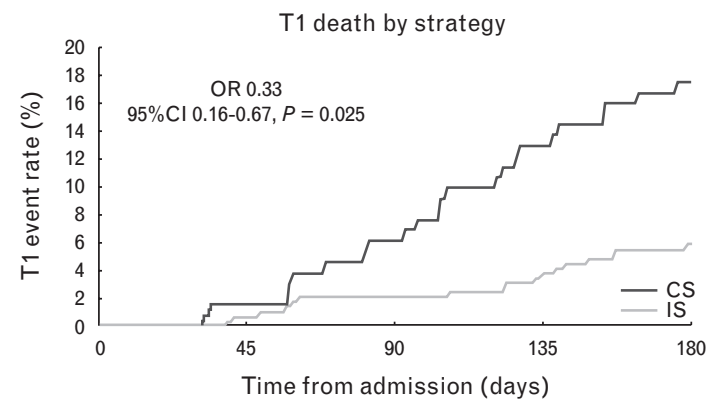

Strategy Number of patients at risk

$\begin{array}{llllll}\text { IS } & 131 & 129 & 123 & 114 & 108 \\ \text { CS } & 290 & 288 & 284 & 279 & 273\end{array}$

(c)

T2 death by strategy

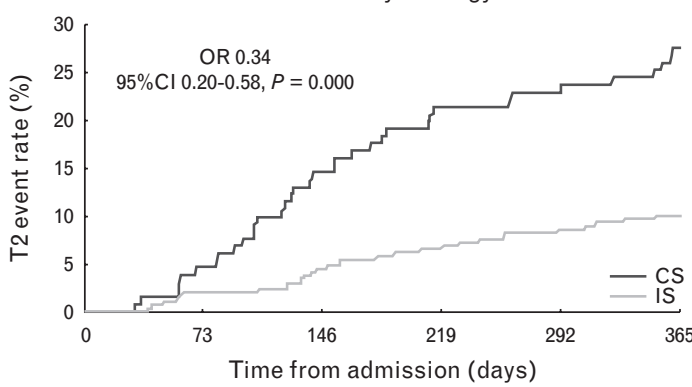

Strategy Number of patients at risk

$\begin{array}{lllllll}\text { IS } & 131 & 125 & 112 & 103 & 100 & 95 \\ \text { CS } & 290 & 284 & 277 & 271 & 265 & 261\end{array}$

(d)

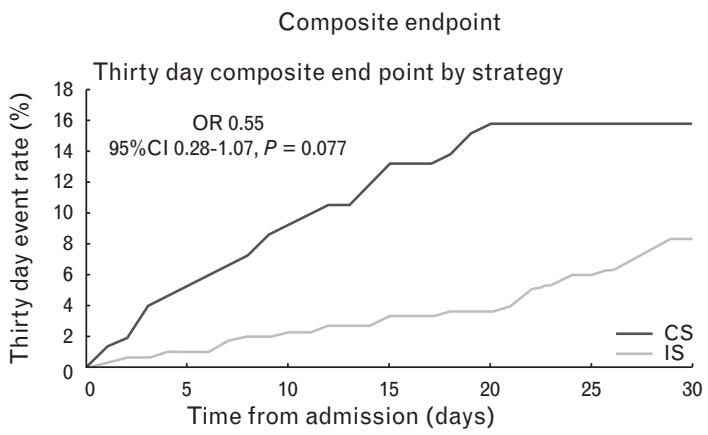

Strategy Number of patients at risk

$\begin{array}{llllllll}\text { IS } & 152 & 144 & 138 & 132 & 128 & 128 & 128 \\ \text { CS } & 301 & 298 & 294 & 291 & 283 & 283 & 276\end{array}$

(e)

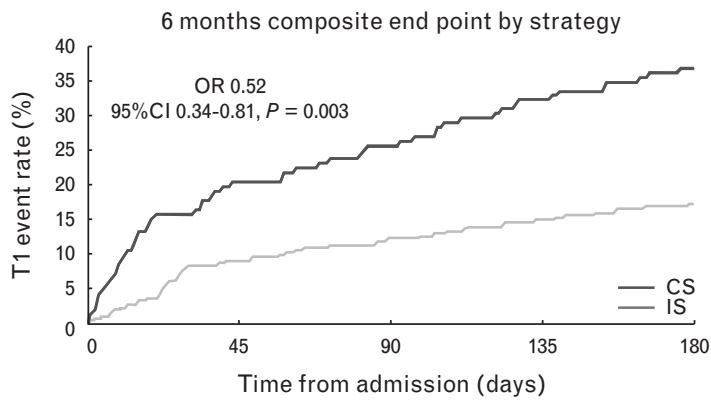

Strategy Number of patients at risk

$\begin{array}{lllllc}\text { IS } & 152 & 121 & 113 & 103 & 96 \\ \text { CS } & 301 & 274 & 264 & 256 & 249\end{array}$

(f)

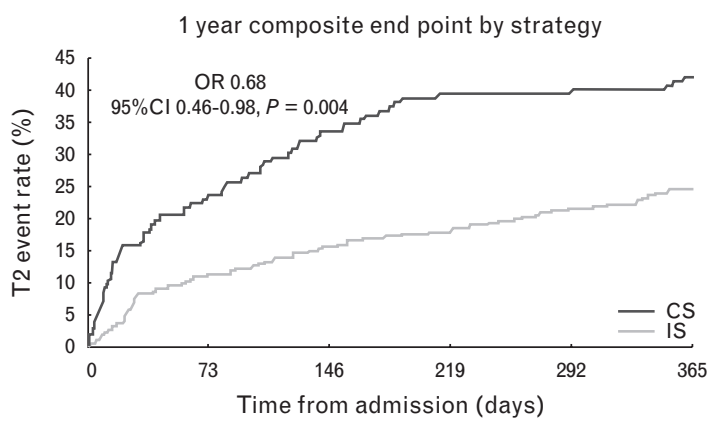

Strategy Number of patients at risk

$\begin{array}{lcccccc}\text { IS } & 152 & 116 & 101 & 92 & 91 & 88 \\ \text { CS } & 301 & 267 & 254 & 246 & 236 & 227\end{array}$

Time to all-cause mortality according to conservative or invasive strategy at 30 days (a), T1 (b) and T2 (c). Time to composite endpoint according to conservative or invasive at 30 days (d), 6 months (e) and 1 year (f).

and very old NSTEACS patients $(75-79,80-84$, older than 85 years, Table 1 ) and the invasive strategy effect on mortality was found at any time. Advanced Killip class was the factor with the strongest effect on in-hospital (OR $8.40,95 \%$ CI $2.03-34.80, P=0.003)$ and 30-day mortality (OR 4.64, 95\% CI 1.45-14.88, $P=0.010)$ and influenced significantly also the mid- (OR $10.05,95 \%$ CI 3.36-30.08, $P<0.0001)$ and the long-term outcome (OR 3.96, 95\% CI
1.86-8.44, $P<0.0001)$. Albumin values greater than $3 \mathrm{~g} / \mathrm{dl}$ have been found protective in mid-term and long-term follow-up and were significantly associated with decreased T1 $(P=0.004)$ and T2 mortality $(P=0.004)$ (Table 3$)$. Of interest, the benefits of an invasive strategy at 1 year were higher in patients with albumin levels of $3 \mathrm{~g} / \mathrm{dl}$ or less, with interaction between albumin and invasive strategy effect on mortality showing 
Table 4 Composite endpoint predictors at the prespecified time points by univariate and multivariate analysis

\begin{tabular}{|c|c|c|c|c|c|c|}
\hline Cumulative composite endpoint & OR univariate & $95 \% \mathrm{Cl}$ & $P$ & OR multivariate & $95 \% \mathrm{Cl}$ & $P$ \\
\hline \multicolumn{7}{|l|}{ 30-Day } \\
\hline Invasive strategy ${ }^{a}$ & 0.48 & $0.27-0.88$ & 0.017 & 0.55 & $0.28-1.07$ & 0.077 \\
\hline Ejection fraction $30-50 \%{ }^{b}$ & 2.04 & $0.92-4.49$ & 0.078 & 1.96 & $0.87-4.41$ & 0.102 \\
\hline Ejection fraction $\leq 30 \%{ }^{\mathrm{b}}$ & 4.57 & $1.89-11.05$ & 0.001 & 3.91 & $1.56-9.80$ & 0.004 \\
\hline \multicolumn{7}{|l|}{ 6-Month } \\
\hline Invasive strategy ${ }^{a}$ & 0.41 & $0.28-0.60$ & $<0.0001$ & 0.52 & $0.34-0.81$ & 0.003 \\
\hline Admission albumin level $>3 \mathrm{~g} / \mathrm{dl}$ & 0.55 & $0.37-0.82$ & 0.004 & 0.57 & $0.38-0.87$ & 0.010 \\
\hline Diabetes & 1.93 & $1.32-2.82$ & 0.001 & 1.74 & $1.16-2.63$ & 0.008 \\
\hline Admission Killip class $3^{\mathrm{C}}$ & 4.21 & $2.48-7.15$ & $<0.0001$ & 3.36 & $1.89-5.90$ & $<0.0001$ \\
\hline Admission Killip class $4^{\mathrm{c}}$ & 24.14 & $3.22-180.76$ & 0.002 & 17.05 & $2.12-137.14$ & 0.008 \\
\hline \multicolumn{7}{|l|}{ 12-Month } \\
\hline Invasive strategy ${ }^{a}$ & 0.47 & $0.36-0.69$ & $<0.0001$ & 0.68 & $0.46-0.98$ & 0.004 \\
\hline Admission systolic blood pressure $(\mathrm{mmHg})$ & 0.99 & $0.99-1.00$ & 0.018 & 0.99 & $0.99-1.00$ & 0.031 \\
\hline Diabetes & 1.77 & $1.27-2.48$ & 0.001 & 1.60 & $1.12-2.28$ & 0.010 \\
\hline Admission creatinine clearance $30-60 \mathrm{ml} / \mathrm{min}^{\mathrm{d}}$ & 1.72 & $1.01-2.93$ & 0.045 & 1.49 & $0.86-2.58$ & 0.152 \\
\hline Admission creatinine clearance $<30 \mathrm{ml} / \mathrm{min}^{\mathrm{d}}$ & 3.41 & $1.96-5.94$ & $<.0001$ & 2.09 & $1.14-3.82$ & 0.017 \\
\hline Admission Killip class $3^{\mathrm{e}}$ & 3.30 & $2.06-5.28$ & $<.0001$ & 2.73 & $1.65-4.52$ & $<0.0001$ \\
\hline
\end{tabular}

$\mathrm{Cl}$, confidence interval; OR, odds ratio. ${ }^{a}$ Conservative strategy as ref. ${ }^{b} \mathrm{EF}>50 \%$ as ref. ${ }^{\mathrm{c}} \mathrm{Killip} 1$ as ref. ${ }^{d}>60 \mathrm{ml} / \mathrm{min}$ as ref. ${ }^{e}$ Killip 1 as ref.

a reinforcement of invasive strategy benefit with albumin levels lower than $3 \mathrm{~g} / \mathrm{dl}$ ( $P$ for interaction 0.037 ).

\section{Composite outcome}

The composite outcome of all-cause mortality and MI occurred in 74 (24.6\%) invasive patients and in $64(42.1 \%)$ conservative patients during 1-year followup $(P<0.0001)$. At 30 days, 6 and 12 months, MIs rates did not differ significantly between the two adopted strategies. Cumulative composite outcome rates at the prespecified time points are reported in Table 2.

Crude and adjusted association between strategy and cumulative composite outcome rate are reported in Table 4. No significant association between strategy and 30-day composite outcome was found. However, invasive patients had lower composite outcome rate at 6 (OR $0.52,95 \%$ CI $0.34-0.81, P=0.003)$ and 12 months (OR $0.68,95 \%$ CI $0.46-0.98, P=0.004$ ) (Fig. $1 \mathrm{E}-\mathrm{F}$ and Table 4). No interaction between the three defined age classes of young old, old and very old NSTEACS patients and invasive strategy effect on composite outcome was found at any time.

Ejection fraction less than 30\%, Killip class, diabetes and advanced kidney disease (creatinine clearance $<30 \mathrm{ml} /$ $\mathrm{min}$ ) were all associated with higher composite outcome rates with statistical significance varying according to follow-up duration (Table 4). Albumin values greater than $3 \mathrm{~g} / \mathrm{dl}$ were associated with significantly lower composite outcome $(P=0.010)$ at 6 months (Table 4). A nonsignificant interaction $(P=0.075)$ was found between albumin and effect of invasive strategy, with early revascularization achieving the most significant reduction of composite outcome at 1 year in patients with albumin $3 \mathrm{~g} / \mathrm{dl}$ or less. Similar results were found using genetic matching approach. No interaction between strategy and age or the specific conditions considered was found.

\section{Discussion}

The present large monocentric study of a consecutive academic cohort of elderly patients with NSTEACS indicates that an invasive strategy provides a consistent reduction in all-cause mortality and composite outcome (all-cause mortality and MI) at the different prespecified time points. Multivariate and propensity score analyses show that in the elderly cohort, an invasive strategy is associated with a three-fold decrease in all-cause mortality, and a two-fold lower rate of composite outcome at short-, mid- or long-term follow-up. In addition, advanced Killip class and a left ventricular ejection fraction lower than $30 \%$ resulted as the main predictors of short-term outcome.

Our study is representative of the high-risk elderly patients admitted with diagnosis of NSTEACS in a contemporary ICCU. About $70 \%$ of our patients were characterized by a GRACE risk score higher than 170, approximately $15 \%$ presenting with advanced Killip class and more than $60 \%$ with a left ventricular EF lower than 50\%. Two-thirds of the overall patients were managed invasively. In European and US registries, the reported proportion of elderly patients assigned to an invasive strategy is slightly lower and declines from 50 to $33 \%$ in patients aged beyond 70 and 80 years, respectively. ${ }^{12-15}$

Data from the present study indicate that the invasively managed patients were characterized by lower age, a typical clinical presentation, a less severe clinical profile, with lower prevalence of advanced Killip class, and higher hemoglobin and creatinine clearance values, as compared with conservative. Nonetheless, despite these differences, the statistical post-hoc adjustment method adopted in our study allowed the balancing of baseline characteristics between the invasive and conservative groups by matching each invasive patient to a conservative one who has the nearest conditional probability of receiving the same treatment, given his/her measured baseline characteristics. 


\section{Treatment strategy and outcome}

Our results show a lower invasive-related mortality and composite outcome risk at either short-, mid- and longterm follow-up, with a sizeable reduction in both endpoints by about two- or three-fold. The mortality benefit of an aggressive strategy has been previously demonstrated in non-ad-hoc designed randomized trials and in large registries, in particular in high-risk elderly patients with ACSs. ${ }^{12-22}$ However, a recent trial, enrolling elderly high-risk NSTEACS patients treated invasively from January 2008 to May 2010, did not face a reduced mortality, but experienced significantly better survival free from the composite of all-cause mortality, nonfatal MI, disabling stroke and repeat hospitalization for cardiovascular causes or bleeding. ${ }^{8}$

In our study, the benefit of an early invasive strategy persists across all age groups, including the old (older than 75 years) and very old (85 years or older), in agreement with the Myocardial Ischemia National Audit Project (MINAP) registry data. ${ }^{23}$ In addition, the troponin positivity status at admission did not affect the mortality benefit of the invasive strategy contrary to what observed by Savonitto et al. ${ }^{7}$

\section{Other predictors of outcome}

Hemodynamic conditions such as a presentation with advanced Killip classes and severely impaired left ventricular EF were the main predictors of the in-hospital phase and short-term outcome. These data appear consistent with those previously published, and individuate hemodynamic instability, as the most relevant predictor of outcome in the elderly patients with NSTEACS during the acute phase of illness. ${ }^{24,25}$

Moreover, normal serum albumin had a protective effect in the mid- and long-term with regard to both mortality and composite outcome. Albumin is considered as a surrogate marker of frailty. ${ }^{26}$ Interestingly, no interaction was found between serum albumin levels and the choice of the treatment strategy and the long-term benefit of invasive management appeared strengthened in patients with low levels of albumin. These data may suggest that frailty should not represent a reason for not referring elderly to invasive treatment. However, the management decision for elderly patients with NSTEACS is actually complex and it should be 'patient centered', as reported in recent guidelines, considering patient preferences, goal, comorbidities, functional and cognitive status and life expectancy. ${ }^{27}$

\section{Limitations}

The present data represent a real-world monocentric registry of elderly NSTEACS treatment at an academic hospital. Individual decisions on patient management such as reasons for not offering an early invasive treatment were not recorded. Hence the issue of residual selection bias leading to confounding cannot be fully excluded despite propensity adjustment and homogeneity of hospital treatment protocols and staffing organization across the years of enrollment. Nevertheless the sample size is adequate to show difference in outcome between the two adopted strategies, and reported data are consistent with that coming from other published studies, both observational ${ }^{20}$ and randomized. ${ }^{21}$ Finally, a prospective evaluation of frailty assessment with measurements of daily living activities was not collected and computed.

\section{Conclusion}

An invasive revascularization strategy offered to elderly patients with NSTEACS was associated with about three-fold improved mortality and composite outcome at either short-, mid- or long-term, in both frail and nonfrail patients. An advanced Killip class and hemodynamic status appear to be the major determinants of outcome during in-hospital phase of NSTEACS. Elderly patients with NSTEACS exhibit a high ischemic risk profile and should be more often offered evidence-based medical therapies and early revascularization.

\section{Acknowledgement}

There are no conflicts of interest.

\section{References}

1 Global Atlas on cardiovascular disease prevention and control. Available at http://whqlibdoc.who.int/publications/2011/9789241564373_eng.pdf.

2 The future of CVD.Mackay J, Mensah G, editors. The atlas of heart disease and stroke. Geneva/Switzerland: World Health Organization; 2004. pp. 74-75.

3 Murray CJ, Lopez AD. Mortality by cause for eight regions of the world: Global Burden of Disease Study. Lancet 1997; 349:1269-1276.

4 Gurwitz JH, Col NF, Avorn J. The exclusion of the elderly and women from clinical trials in acute myocardial infarction. JAMA 1992; 268:1417-1422.

5 Avezum A, Makdisse M, Spencer F, et al., GRACE Investigators. Impact of age on management and outcome of acute coronary syndrome: observations from the global registry of acute coronary events (GRACE). Am Heart J 2005; 149:67-73.

6 Angeli F, Verdecchia P, Savonitto S, et al. Early invasive versus selectively invasive strategy in patients with non-ST-segment elevation acute coronary syndrome: impact of age. Cathet Cardiovasc Intv 2014; 83:686-701.

7 Savonitto S, Cavallini C, Petronio AS, et al., Italian Elderly ACS Trial Investigators. Early aggressive versus initially conservative treatment in elderly patients with non-ST-segment elevation acute coronary syndrome a randomized controlled trial. J Am Coll Cardiol Intv 2012; 5:906-916.

8 Galasso G, De Servi S, Savonitto S, et al. Effect of an invasive strategy on outcome in patients $\geq 75$ years of age with non-ST-elevation acute coronary syndrome. Am J Cardiol 2015; 115:576-580.

9 Plakht $\mathrm{Y}$, Shiyovich A, Gilutz H. Predictors of long-term (10 year) mortality postmyocardial infarction: age-related differences. Soroka acute myocardial infarction (SAMI) project. J Cardiol 2015; 65:216-223.

10 O'Brien E, Subherwal S, Roe MT, et al. Do patients treated at academic hospitals have better longitudinal outcomes after admission for non-ST elevation myocardial infarction? Am Heart J 2014; 167:762-769.

11 Bassand JP, Hamm CW, Ardissino D, et al. Task Force for Diagnosis and Treatment of Non-ST-Segment Elevation Acute Coronary Syndromes of European Society of Cardiology. Guidelines for the diagnosis and treatment of non-ST-segment elevation acute coronary syndromes. Eur Heart J 2007; 28:1598-1660.

12 Devlin G, Gore JM, Elliott J, et al., GRACE Investigators. Management and 6-month outcomes in elderly and very elderly patients with high-risk non-STelevation acute coronary syndromes: The Global Registry of Acute Coronary Events. Eur Heart J 2008; 29:1275-1282.

13 Granger CB, Goldberg RJ, Dabbous O, et al., Global Registry of Acute Coronary Events Investigators. Predictors of hospital mortality in the Global Registry of Acute Coronary Events. Arch Intern Med 2003; 163:2345-2353. 
14 Bach RG, Cannon CP, Weintraub WS, et al. The effect of routine, early invasive management on outcome for elderly patients with non-ST-segment elevation acute coronary syndromes. Ann Intern Med 2004; 141:186-195.

15 Van Diepen S, Chen AY, Wang TY, et al. Influence of heart failure symptoms and ejection fraction on short- and long-term outcomes for older patients with non-ST-segment elevation myocardial infarction. Am Heart $J$ $2014 ; 167: 267-273$.

16 Bagnall AJ. Influence of age on use of cardiac catheterization. Am J Cardiol 2009; 103:1530-1536.

17 Buber J, Goldenberg I, Kimron L, et al. One year outcome following coronary angiography in elderly patients with non-ST elevation myocardial infarction: real-world data from the Acute Coronary Syndromes Israeli Survey (ACSIS). Coronary Artery Dis 2013; 24:102-109.

18 Kuch B, Wende R, Barac M, et al. Prognosis and outcomes of elderly (7584 years) patients with acute myocardial infarction 1-2 years after the event: AMl-elderly study of the MONICA/KORA Myocardial Infarction Registry. Int J Cardiol 2011; 149:205-210.

19 Shirasawa K, Hwang MW, Sasaki Y, et al. Mortality and changes in physical ability after coronary revascularization for octa-nonagenarian patients with acute coronary syndrome. Heart Vessels 2011; 26:385-391.

20 McAlister FA, Oreopoulos A, Norris CM, et al., Alberta Provincial Project for Outcome Assessment in Coronary Heart Disease (APPROACH) Investigators. Exploring the treatment-risk paradox in coronary disease. Arch Intern Med 2007; 167:1019-1025.

21 Malkin CJ, Prakash R, Chew DP. The impact of increased age on outcome from a strategy of early invasive management and revascularisation in patients with acute coronary syndromes: retrospective analysis study from the ACACIA registry. BMJ Open 2012; 2:e000540.
22 Damman P, Clayton T, Wallentin L, et al. Effects of age on longterm outcomes after a routine invasive or selective invasive strategy in patients presenting with non-ST segment elevation acute coronary syndromes: a collaborative analysis of individual data from the FRISC II - ICTUS - RITA-3 (FIR) trials. Heart 2012; 98:207213.

23 Gale CP, Cattle BA, Woolston A, et al. Resolving inequalities in care? Reduced mortality in the elderly after acute coronary syndromes. The Myocardial Ischaemia National Audit Project 2003-2010. Eur Heart J 2012; 33:630-639.

24 Vervueren PL, Elbaz M, Wagner A, et al. The major element of 1-year prognosis in acute coronary syndromes is severity of initial clinical presentation: results from the French MONICA registries. Arch Cardiovasc Dis 2012; 105:478-488.

25 Velazquez EJ, Francis GS, Armstrong PW, et al., VALIANT Registry. An international perspective on heart failure and left ventricular systolic dysfunction complicating myocardial infarction: the VALIANT registry. Eur Heart J 2004; 25:1911-1919.

26 Robinson TN, Eiseman B, Wallace Jl, et al. Redefining geriatric preoperative assessment using frailty, disability and co-morbidity. Ann Surg 2009; 250:449-455.

27 Amsterdam EA, Wenger NK, Brindis RJ, et al. 2014 AHA/ACC guidelines for the management of patients with non-ST-elevation acute coronary syndromes: executive summary: a report of the American College of Cardiology/American Heart Association Task Force on Practice Guidelines. J Am Coll Cardiol 2014; 64:26452687. 